\title{
An Unusual Case of Corpulmonale Due to a Common Iliac Artery Aneurysm Fistulating to the Common Iliac Vein
}

\author{
Nisheeth Kansala, Reem Salman ${ }^{\mathrm{a}}$, Colin Nice ${ }^{\mathrm{a}}$, Vish Bhattacharya ${ }^{\mathrm{a}, \mathrm{b}}$
}

\begin{abstract}
This is an unusual case presenting as bilateral lower limb edema and corpulmonale secondary to left ilio-ilial fistula. A 70-year-old man with a background history of hypertension and ischemic heart disease presented to the medical team with bilateral leg swelling and deranged liver and kidney function. An abdominal ultrasound scan detected an aneurysm of the left common iliac artery. A CT angiogram revealed that this aneurysm was fistulating into the left common iliac vein causing right heart failure. This was repaired endovascularly. The procedure was successful and resulted in complete resolution of symptoms and restoration of normal liver function.
\end{abstract}

Keywords: Ilio-ilial fistula; Aneurysm; Right heart failure

\section{Introduction}

We present a rare case of a fistula from an iliac artery aneurysm into the iliac vein causing right heart failure.

This was treated successfully with an iliac covered stent resulting in closure of the fistula and complete resolution of heart failure and liver and renal functions.

This is an extremely rare case of right heart failure due to volume overload from a fistula from a left iliac aneurysm.

\section{Case Report}

A 70-year-old gentleman with a background history of hypertension, ischemic heart disease and high BMI presented as an emergency to the accident and emergency department with increased shortness of breath, cough and bilateral lower limb

Manuscript accepted for publication November 20, 2015

aDepartment of Vascular and General Surgery, Queen Elizabeth Hospital, Gateshead NE96SX, UK

bCorresponding Author: Vish Bhattacharya, Queen Elizabeth Hospital, Gateshead NE96SX, UK. Email: vbhatta@yahoo.com

doi: http://dx.doi.org/10.14740/jmc2375w peripheral edema.

His ECG demonstrated atrial flutter and ST depression of the anterolateral leads which gave the impression of an atypical non-ST-elevation myocardial infarction (NSTEMI). His chest examination revealed mild basal crackles worse on the left side and his abdominal examination revealed an enlarged liver. Bloods revealed that his troponin $\mathrm{T}$ and $\mathrm{CRP}$ were elevated all of which supported the diagnosis of NSTEMI.

He received diuretics as well as bisoprolol for rate control but he deteriorated further as he was nauseous, vomiting and dropping oxygen saturation. His liver and renal function tests were deranged and suggested features of congestive liver failure. His inflammatory markers and clotting time were elevated.

\section{Investigations}

An ultrasound revealed a left common iliac artery aneurysm that measured 61 (antero-posterior) $\times 82 \mathrm{~mm}$ (transverse) diameter with appearances due to a false saccular aneurysm (Fig. 1).

It also showed that the inferior vena cava (IVC) was enlarged (26 $\mathrm{mm}$ in antero-posterior diameter) and remained dilated throughout the respiratory cycle, appearances suggestive of congestive cardiac failure.

A CT angiogram showed an $8 \mathrm{~cm}$ saccular aneurysm left

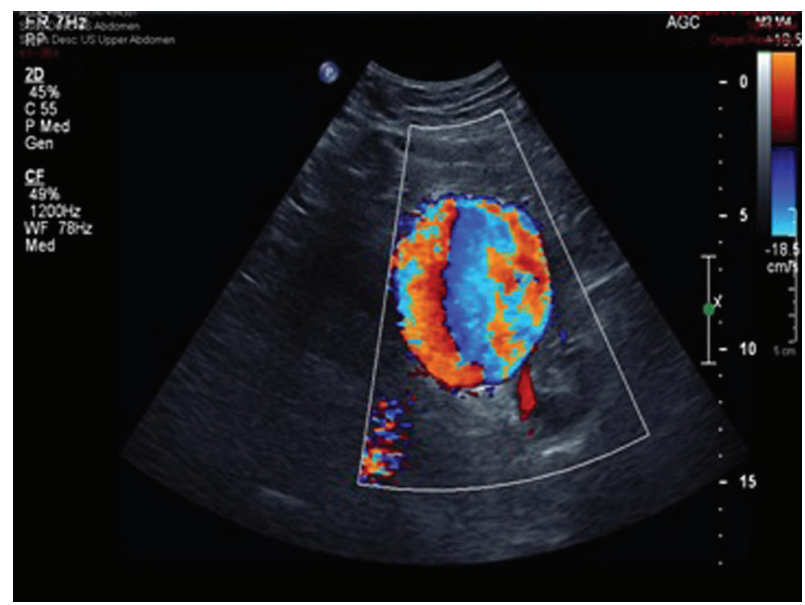

Figure 1. Color Doppler showing both arterial and venous flow within the aneurysm sac. 


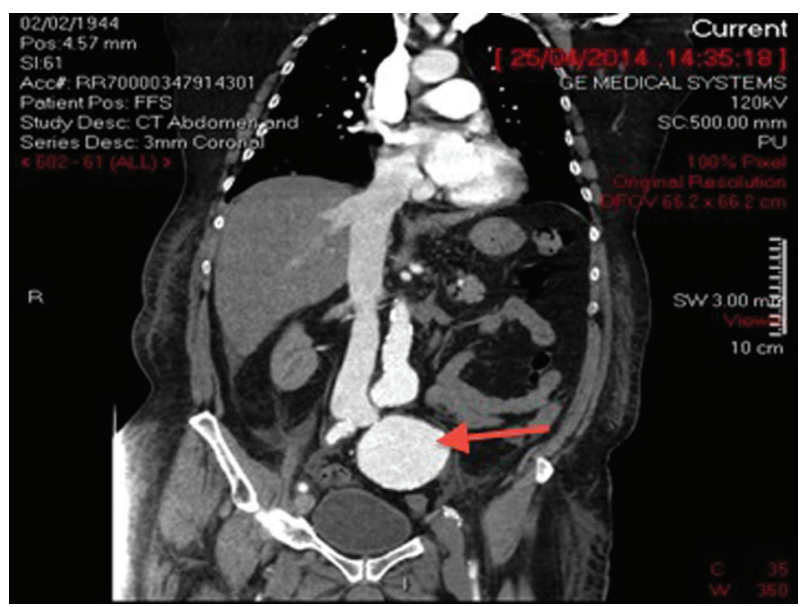

Figure 2. Large left iliac artery aneurysm with fistula into the left iliac vein.

common iliac artery with an associated arterio-venous fistula at the level of the left common iliac vein (Fig. 2, 3).

\section{Treatment}

An endovascular stenting approach was recommended after discussion at the multidisciplinary meeting. The procedure was done via standard open exposure of the left groin. A 7-French catheter was inserted percutaneously in the right femoral artery followed by a guide wire which passed over the bifurcation into left iliac artery.

A Terumo wire was passed up the right sheath and retrieved via the left sheath, thus enabling straightening of the iliac vessels and passage of catheter into aorta. A Lunderquist wire was passed into the aorta via the left groin. An attempt was made to embolize the left internal iliac artery but access proved impossible due to the angulation and the thrombus in the common iliac artery.

A Cook Zenith stent ${ }^{\circledR}(13 \times 107 \mathrm{~mm})$ was then deployed successfully (Fig. 4).

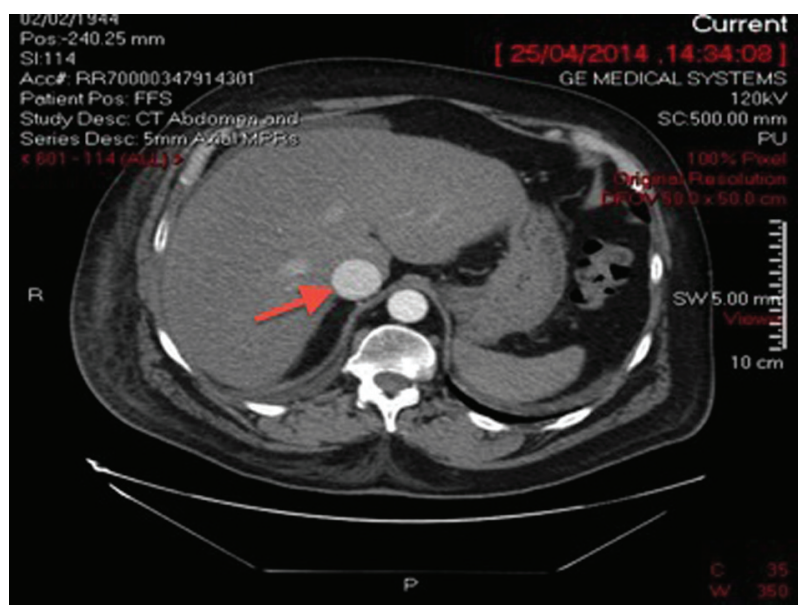

Figure 3. Inferior vena cava showing contrast flow due to fistula connection with iliac aneurysm.

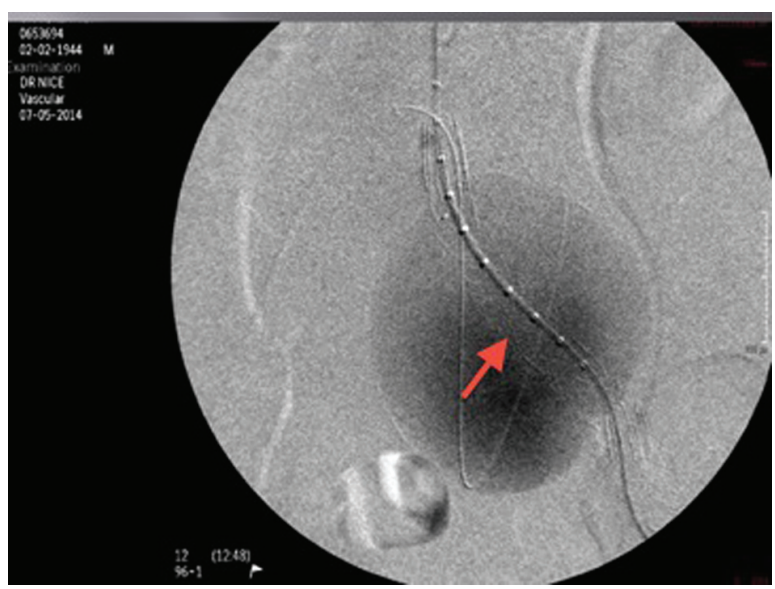

Figure 4. Covered Cook Zenith stent in place in left common iliac artery.

\section{Outcome and follow-up}

He was admitted to the high dependency unit (HDU) following the procedure where he made good recovery and was then transferred to a surgical ward. His leg swelling improved gradually. His liver as well as renal function tests improved rapidly and gradually came to normal.

Follow-up scans at 8 weeks (Fig. 5, 6) revealed exclusion of fistula and minimal flow of contrast in the IVC.

Clinically, there was complete resolution of symptoms and he was able to return to normal activity (Fig. 7).

\section{Discussion}

Spontaneous arteriovenous fistula causing right heart failure from the common iliac artery to the iliac vein has never been reported to the best of our knowledge.

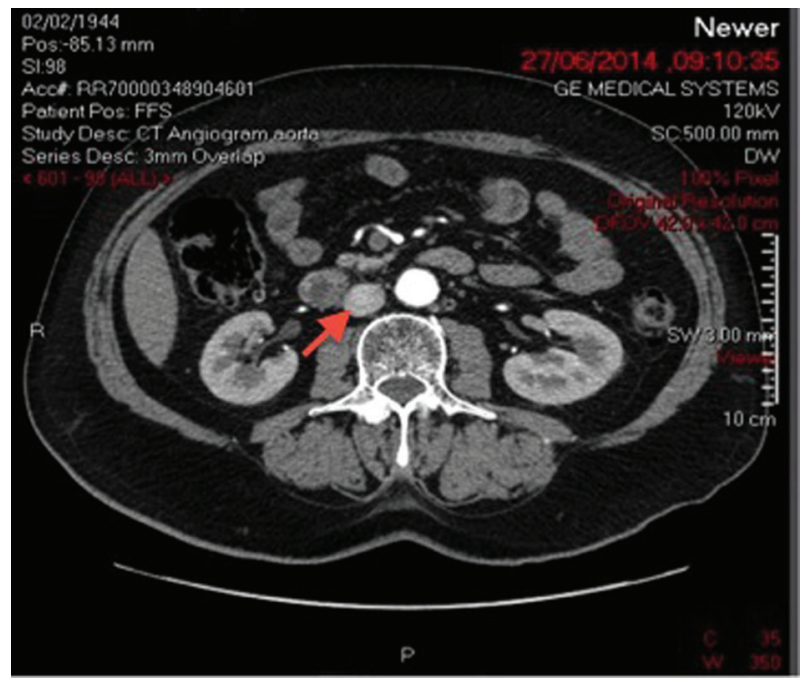

Figure 5. Post-operative images showing no contrast in IVC due to successful closure of fistula. 


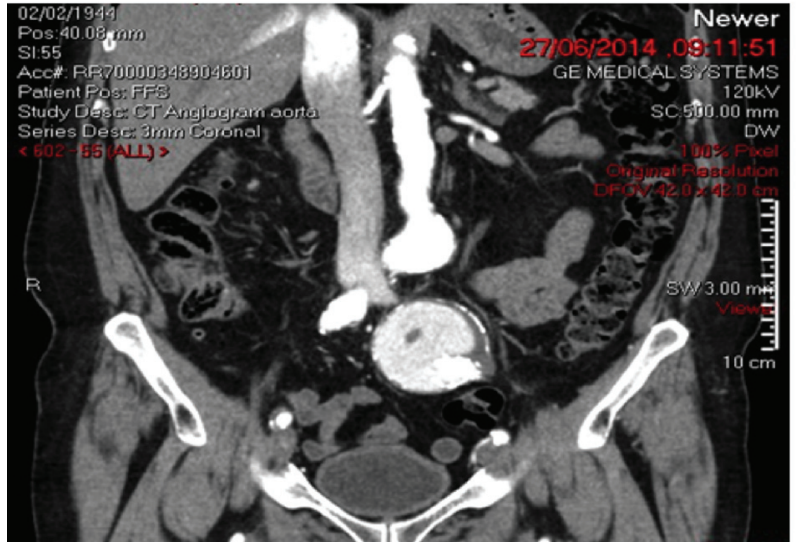

Figure 6. Post-operative images showing exclusion of fistula and no contrast in IVC.

Aortocaval fistula is an uncommon complication of large abdominal aortic aneurysms (AAAs) and can occur with or without retroperitoneal rupture. A high index of clinical suspicion along with prompt diagnosis and surgical treatment is of outmost importance in the management of such a condition. The literature search reports two cases of open repair [1].

Over $80 \%$ of reported aortocaval fistulas are related with ruptured AAAs. Other causes refer to penetrating trauma, mycotic aneurysms, Takayasu's arteritis, and connective tissue diseases [2].

Rupture into the vena cava may be asymptomatic and recognized during elective AAA repair or may be overlooked when symptoms of rupture predominate. However, preoperative diagnosis is essential, in order to minimize blood loss and avoid possible intraoperative pulmonary embolism $[3,4]$.

A few reports have been published over the past years regarding successful endovascular treatment of aortocaval fistulation.

Although most of them report favorable results concern- ing early survival and short hospitalization, persistent type II endoleak has been noted, while long-term follow-up is missing $[5,6]$.

A typical clinical presentation includes sudden onset of abdominal pain, shortness of breath, and a pulsatile abdominal mass with an audible machinery-like bruit and/or a thrill [7].

Nevertheless, symptoms seem to be related with the hemodynamics of the communication. In large, high-flow aortocaval fistulas, symptoms of cardiac failure, and sudden central venous hypertension with no clear cause may be the only findings suggesting the diagnosis [8].

Despite the low frequency of arteriovenous fistulae in aorto-iliac aneurysms, a careful physical examination should raise clinical suspicion. An abdominal "murmur" associated with pain and abdominal mass on palpation may be present [9].

\section{Conclusion}

We have presented a rare case of an iliac aneurysm fistulating into the left iliac vein causing right heart failure. Stenting of the aneurysm resulted in complete alleviation of symptoms. This represents an unusual cause of right heart failure and should be considered in the differential diagnosis of this medical condition.

\section{Disclaimer}

A formal ethical approval was granted for this study and written consent was obtained from this patient.

\section{References}

1. Psathas ED, Lioudaki S, Doulaptsis M, Charalampoudis

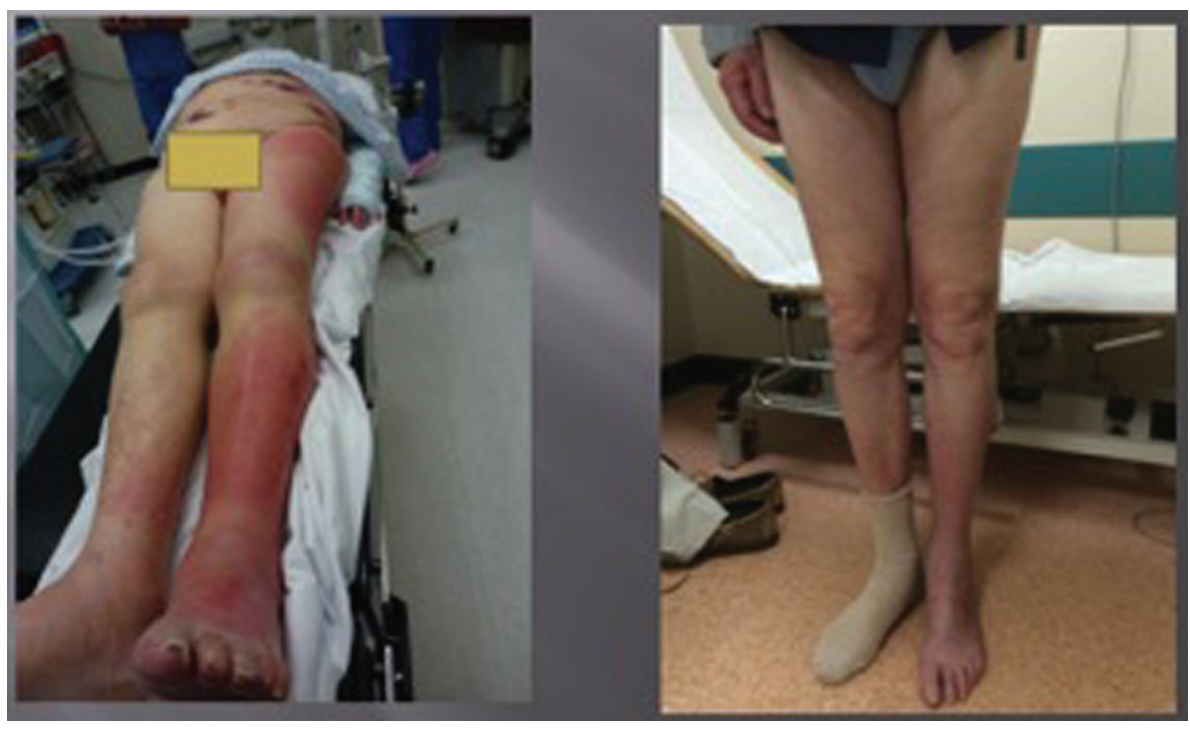

Figure 7. Pre- and post-operative appearance of legs following successful stenting of aneurysm and exclusion of fistula resulting in complete resolution of leg swelling. 
P, Klonaris C, Verikokos C. Clinical manifestations of aortocaval fistulas in ruptured abdominal aortic aneurysm: report of two cases. Case Rep Surg. 2012;2012:123081.

2. Bednarkiewicz M, Pretre R, Kalangos A, Khatchatourian G, Bruschweiler I, Faidutti B. Aortocaval fistula associated with abdominal aortic aneurysm: a diagnostic challenge. Ann Vasc Surg. 1997;11(5):464-466.

3. Davis PM, Gloviczki P, Cherry KJ, Jr., Toomey BJ, Stanson AW, Bower TC, Hallett JW, Jr. Aorto-caval and ilio-iliac arteriovenous fistulae. Am J Surg. 1998;176(2):115-118.

4. Weigent CE. Pulmonary atheroembolism complicating repair of an atherosclerotic abdominal aneurysm. Minn Med. 1978;61(1):15-16.

5. Akwei S, AltafN, Tennant W, MacSweeney S, Braithwaite B. Emergency endovascular repair of aortocaval fistula- -a single center experience. Vasc Endovascular Surg. 2011;45(5):442-446.

6. Vetrhus M, McWilliams R, Tan CK, Brennan J, GillingSmith G, Harris PL. Endovascular repair of abdominal aortic aneurysms with aortocaval fistula. Eur J Vasc Endovasc Surg. 2005;30(6):640-643.

7. Lin PH, Bush RL, Lumsden AB. Aortocaval fistula. J Vasc Surg. 2004;39(1):266.

8. Leigh-Smith S, Smith RC. Aorto caval fistula--the "bursting heart syndrome". J Accid Emerg Med. 2000;17(3):223225.

9. Daniel Mendes PintoI, Leonardo Ghizoni BezI, Jose Olimpio Dias JuniorII, Caetano de Sousa LopesI, Ari MandilI. lliac aneurysm associated with arteriovenous fistula. J Vasc Bras. 2007;6(3):297-300. 